\title{
An emergence of Mycobacterium abscessus in blood cultures of Thai patients
}

\author{
Duangjai Boonlert, Phitchanan Poosanga, Suporn Foongladda, Piriyaporn Chongtrakool and Popchai \\ Ngamskulrungroj *
}

\author{
Department of Microbiology, Faculty of Medicine Siriraj Hospital, Mahidol University, Bangkok, Thailand. \\ Email: popchai.nga@mahidol.ac.th
}

Received 14 March 2019; Received in revised form 12 September 2019; Accepted 3 October 2019

\begin{abstract}
Aims: Tuberculosis and other mycobacterial infections occur worldwide especially in patients with immunodeficiency. Typically, an empirical treatment for disseminated disease is required for initial therapy due to slow growing nature of most mycobacterial species. Therefore, species distribution and average time to positivity of blood culture is crucial. However, such information is limited for blood culture and, therefore, were determined.

Methodology and results: The blood culture data using the BACTEC FX system and drugs susceptibility testing (DST) pattern was recovered during 2012-2017 from a large teaching hospital in Bangkok, Thailand. Overall, $7.8 \%$ of 4,838 blood and $6.4 \%$ of 1,056 bone marrow (BM) samples were positive for mycobacterial growth. Mycobacterium tuberculosis complex (MTBC), M. avium, and M. abscessus, were the most three common species to be isolated from blood (3.8\%, $2.1 \%$, and $0.9 \%$, respectively) and $\mathrm{BM}(2.4 \%, 2.4 \%$, and $0.9 \%$, respectively). The average time to positivity for MTBC, M. avium, and M. abscessus was 25.7, 16.1, and 3.8 days, respectively. From 209 antimycobacterial susceptibility testing (AST)-available MTBC strains, $6(2.87 \%)$ strains were multi-drugs resistant (MDR-TB). From 35 AST-available M. avium complex (MAC) isolates, $6(17.14 \%), 33(94.29 \%)$, and $28(80 \%)$ isolates were resistant to clarithromycin, moxifloxacin, and linezolid, respectively. BM MAC isolates were significantly more resistant to clarithromycin than the blood isolates (44.5\% vs $7.69 \% ; p=0.027)$.

Conclusion, significance and impact of study: In summary, an emergence of $M$. abscessus and unusually high moxifloxacin and linezolid resistance of MAC isolates were reported in this study. Additional information of this study benefits physicians for anti-mycobacterial drug selection for initial treatment of mycobacteremia while blood and BM culture is pending.
\end{abstract}

Keywords: Mycobacterium abscessus, blood culture, bone marrow, detection time, susceptibility testing

\section{INTRODUCTION}

Mycobacteria, is an acid-fast, Gram-positive, rod-shaped bacteria and is classified into two main groups, the slow and rapid grower (Gangadharam and Jenkins, 1997). The slow grower includes Mycobacterium tuberculosis complex (MTBC) and most species of non-tuberculous mycobacteria (NTM), such as $M$. avium complex (MAC). The rapid grower includes $M$. abscessus, $M$. fortuitum complex and $M$. chelonae complex. MTBC, a causative agent of tuberculosis, is primarily transmitted via aerosol droplets and commonly causes respiratory diseases (Kent, 1985).

Over the past decade, there has been a significant increase in reports of disseminated mycobacterial infection in immunocompromised patients (Bouza et al., 1988). MAC is the most common cause of systemic mycobacterial infection in immunocompromised patients. The increasing trend of NTM infections especially in immunocompromised patients, leads to the need of effectively identifying the etiologic agent for selecting the most appropriate treatment regimen (Chin et al., 1994).

Due to the increased prevalence of systemic mycobacterial infections, several reports of mycobacteremia were published recently. For example, a previous study demonstrated detections of mycobacteria in the bloodstream of AIDS patients in a University Hospital in Brazil in 2001. With a total of 530 mycobacterial blood cultures incubated in the BACTEC 460 TB system, 41 (16\%) patients were positive for mycobacteria. MTBC was recovered in 15 out of 41 $(36.6 \%)$ patients and the average time to positivity of blood culture was 24 days. MAC was recovered in 22 out of $41(53.6 \%)$ patients and the average time to positivity of blood culture was 15 days. Other species of mycobacteria were recovered in 4 out of 41 (9.8\%) patients (Oplustil et al., 2001). In Thailand, a surveillance for bloodstream infections in two rural Thailand provinces 
by the BacT/Alert MB system during 2005-2012 showed $78 \%$ and $22 \%$ of 354 mycobacterial isolates were identified as MTBC and NTM respectively (Jorakate et al., 2015). In 2009, another report of 327 mycobacterial blood isolates from our hospital, Siriraj Hospital in Bangkok, showed MTBC (54.5\%), M. avium (37.5\%), and $M$. intracellulare was the most common (Foongladda et al., 2009). No rapid grower was identified and the average time to detect positive of blood culture was not determined.

For an optimal treatment, species identification of mycobacteria, and antimycobacterial susceptibility are crucial. However, due to the long incubation time of most mycobacterial species, selection of definitive antibiotic is often delayed. The average time to detect positive blood culture of each mycobacterial species can be provisionally used to differentiate the slow grower from the rapid grower. However, information of the average time to detect positive of blood culture is lacking. Therefore, this study aimed to report the average time to detect positive of blood and bone marrow culture of each mycobacterial species. Species distribution and antibiotics susceptibility were also described.

\section{MATERIALS AND METHODS}

\section{Mycobacterial strains, species identification, and detection time}

All mycobacteria strains from blood and bone marrow (BM) cultures were identified in the Mycobacteriology Laboratory, Department of Microbiology, Faculty of Medicine Siriraj Hospital, Mahidol University, during the period 2012-2017. The BACTEC FX System was used according to a manufacturer's instructions (Fuller et al., 2001). The BACTEC Myco/F Lytic media was used. Time from an insertion of the bottle into the instrument until instrument detected positive were also calculated as the time to detect positive. Species identification was confirmed by using in-house real time PCR and Line probe assays according to previous published methods (Tortoli et al., 2003, Foongladda et al., 2009). The time to detect positive were available only in 2016-2017 and only mycobacterial species with least 3 strains were included in the calculation.

\section{Antimycobacterial susceptibility testing}

The antimycobacterial susceptibility testing (AST) for $M$. tuberculosis complex was performed by using [3- $(4,5-$ dimethylthiazol-2-yl)-2,5-diphenyltetrazolium bromide] (MTT) assays (Foongladda et al., 2002), and the automated drug susceptibility using BACTEC ${ }^{\text {TM }}$ MGIT 960 systems (Becton Dickinson, Sparks, MD, USA) (Scarparo et al., 2004), according to the manufacturer's instructions. AST of MAC was performed by micro broth dilution using the TREK Diagnostic Systems (Thermo scientific, West Sussex, UK.), according to the manufacturer's instruction. Interpretation of susceptibilities were done according to the Clinical and Laboratory Standards Institute (CLSI) standard for MGIT and according to the previous publication for MTT (Foongladda et al., 2002). For MTBC, clinical break points for each drug were as follows: isoniazid, resistant, $\geq 0.1 \mu \mathrm{g} / \mathrm{mL}$ (MGIT 960) or $\geq 0.2 \mu \mathrm{g} / \mathrm{mL}$ (MTT); rifampicin, resistant, $\geq 1 \mu \mathrm{g} / \mathrm{mL}$ (both methods); and ethambutol, resistant $\geq 5 \mu \mathrm{g} / \mathrm{mL}$ (both methods). Except isoniazid resistant strains determined by the MGIT 960 , all resistance strains were again confirmed by the agar proportional method according to the CLSI guideline (isoniazid, resistant, $\geq 0.2 \mu \mathrm{g} / \mathrm{mL}$; rifampicin, resistant, $\geq 1$ $\mu \mathrm{g} / \mathrm{mL}$; and ethambutol, resistant $\geq 5 \mu \mathrm{g} / \mathrm{mL}$ ). For MAC, clinical breakpoints were interpreted only for clarithromycin, moxifloxacin and linezolid with cut-off points (CLSI, 2011), as follows: clarithromycin, susceptible, $\leq 8 \mu \mathrm{g} / \mathrm{mL}$; intermediate, $16 \mu \mathrm{g} / \mathrm{mL}$; resistant, $\geq 32 \mu \mathrm{g} / \mathrm{mL}$; moxifloxacin, susceptible, $\leq 1 \mu \mathrm{g} / \mathrm{mL}$; intermediate, $2 \mu \mathrm{g} / \mathrm{mL}$; resistant, $\geq 4 \mu \mathrm{g} / \mathrm{mL}$; and linezolid, susceptible, $\leq 8 \mu \mathrm{g} / \mathrm{mL}$; intermediate, $16 \mu \mathrm{g} / \mathrm{mL}$; resistant, $\geq 32 \mu \mathrm{g} / \mathrm{mL}$. This study was approved by Siriraj hospital ethic committee (Si 765/2017).

\section{Statistical analysis}

Statistical analysis using mean, percentage, and t-test were calculated by Microsoft Excel enterprise 2007 (Microsoft Corporation, Redmond, WA, USA). Significance were defined as $p \leq 0.05$.

\section{RESULTS}

During 2012-2017, 4,838 blood and 1,056 BM samples from patients suspected to have mycobacterial infection, were sent for mycobacterial cultures. $7.8 \%$ of the blood and $6.4 \%$ of the BM samples were positive for mycobacterial growth (Table 1). MTBC and $M$. avium were the most common mycobacterial species to be isolated from both blood (3.8\% and 2.1\%) and BM $(2.4 \%$ and $2.4 \%$ ). In addition, $M$. abscessus was the most common rapid growers isolated from blood $(0.9 \%)$ and BM $(0.9 \%)$, respectively. Distributions of other mycobacterial species were presented in Table 1.

Time to positivity for the mycobacteria from blood and BM culture were then evaluated. The average time to positivity for MTBC was 25.7 days, ranging from 8 to 38 days. For $M$. avium, the average time to positivity was 16.1 days, ranging from 8 to 38 days. While the average time to positivity of $M$. abscessus, a rapid grower, was 3.8 days, ranged from 3 to 7 days. Time to positivity for other mycobacterial species were indicated in Table 2.

From a total of 209 MTBC strains, $6(2.87 \%)$ strains were multi-drugs resistant (MDR-TB) while 23 (11\%) strains and $8(3.82 \%)$ strains were monoresistant to isoniazid and rifampicin, respectively. 184 (88.04\%) strains were pan-susceptible to isoniazid, rifampicin and ethambutol. Percentages of susceptibility of each drug were shown in Table 3. No statistical difference in susceptibility profile between isolates from blood and BM isolates (Table 3 ).

A total of $35 \mathrm{M}$. avium complex isolates were investigated for susceptibility profile using micro broth 
Malays. J. Microbiol. Vol 16(2) 2020, pp. 111-116

DOI: http://dx.doi.org/10.21161/mjm.190399

Table 1: Number of mycobacterial isolates from blood and BM during 2012-2017.

\begin{tabular}{|c|c|c|c|c|c|c|c|c|c|c|c|c|c|c|}
\hline Year & 20 & & & & & & 20 & & 201 & & & & & \\
\hline Sample type & $\mathrm{Bl}$ & $\mathrm{BM}$ & $\mathrm{BI}$ & $\mathrm{BM}$ & $\mathrm{BI}$ & $\mathrm{BM}$ & $\mathrm{Bl}$ & $\mathrm{BM}$ & $\mathrm{BI}$ & $\mathrm{BM}$ & $\mathrm{BI}$ & BM & Blood (\%) & $\mathrm{BM}(\%)$ \\
\hline Total No. of sample & 989 & 245 & 925 & 76 & 816 & 126 & 808 & 238 & 826 & 150 & 474 & 221 & $4,838(100)$ & $1,056(100)$ \\
\hline $\begin{array}{l}\text { Positive for } \\
\text { mycobacteria }\end{array}$ & 87 & 14 & 99 & 10 & 56 & 14 & 58 & 7 & 49 & 15 & 29 & 8 & $378(7.8)$ & $68(6.4)$ \\
\hline M. tuberculosis complex & 48 & 6 & 45 & 4 & 23 & 7 & 32 & 2 & 22 & 5 & 14 & 1 & $184(3.8)$ & $25(2.4)$ \\
\hline - M. avium & 29 & 3 & 19 & 4 & 20 & 5 & 17 & 2 & 13 & 7 & 5 & 4 & $103(2.1)$ & $25(2.4)$ \\
\hline - M. intracellulare & 0 & 1 & 0 & 0 & 0 & 0 & 3 & 1 & 2 & 0 & 0 & 0 & $5(0.1)$ & $2(0.2)$ \\
\hline $\begin{array}{l}\text { - M. avium complex } \\
\text { (other) }\end{array}$ & 2 & 1 & 4 & 0 & 4 & 0 & 4 & 2 & 2 & 3 & 3 & 0 & $19(0.4)$ & $6(0.6)$ \\
\hline - M. simiae & 1 & 0 & 0 & 0 & 2 & 0 & 0 & 0 & 1 & 0 & 0 & 0 & $4(0.1)$ & $0(0)$ \\
\hline - M. scrofulaceum & 0 & 0 & 0 & 1 & 2 & 1 & 1 & 0 & 3 & 0 & 0 & 0 & $6(0.1)$ & $2(0.2)$ \\
\hline - M. kansasii & 0 & 0 & 0 & 1 & 1 & 1 & 0 & 0 & 0 & 0 & 0 & 0 & $1(<0.1)$ & $2(0.2)$ \\
\hline - M. haemophilum & 2 & 0 & 1 & 0 & 0 & 0 & 0 & 0 & 1 & 0 & 2 & 1 & $6(0.1)$ & $1(0.1)$ \\
\hline - M. abscessus & 4 & 3 & 24 & 0 & 4 & 0 & 1 & 0 & 5 & 0 & 4 & 1 & $42(0.9)$ & $4(0.9)$ \\
\hline - M. fortuitum complex & 1 & 0 & 0 & 0 & 0 & 0 & 0 & 0 & 0 & 0 & 0 & 0 & $1(<0.1)$ & $0(0)$ \\
\hline $\begin{array}{l}\text { - other Mycobacterium } \\
\text { spp. }\end{array}$ & 0 & 0 & 6 & 0 & 0 & 0 & 0 & 0 & 0 & 0 & 1 & 1 & $7(0.1)$ & $1(0.1)$ \\
\hline $\begin{array}{l}\text { Negative for } \\
\text { mycobacteria }\end{array}$ & 902 & 231 & 826 & 66 & 760 & 112 & 750 & 231 & 777 & 135 & 445 & 213 & 4460 & 988 \\
\hline
\end{tabular}

$\mathrm{BI}$, blood; $\mathrm{BM}$, bone marrow 
Malays. J. Microbiol. Vol 16(2) 2020, pp. 111-116

DOI: http://dx.doi.org/10.21161/mjm.190399

Table 2: Average time to positivity of each mycobacterial species during 2016-2017 $(n=101)$.

\begin{tabular}{|c|c|c|c|c|c|}
\hline \multirow[t]{2}{*}{ Mycobacterial species } & \multicolumn{2}{|c|}{ No. of isolates } & \multicolumn{2}{|c|}{$\begin{array}{l}\text { Average time to positivity } \\
\text { in days (SD) }\end{array}$} & \multirow[t]{2}{*}{$P$-value } \\
\hline & Blood & BM & Blood & BM & \\
\hline M. tuberculosis complex & 36 & 6 & $26.4(8.75)$ & $27.4(5.03)$ & 0.789 \\
\hline M. avium & 18 & 11 & $17.38(9.64)$ & $20.29(12.43)$ & 0.486 \\
\hline M. avium complex (other) & 5 & 3 & $19(2.58)$ & $16(1.41)$ & 0.119 \\
\hline M. scrofulaceum & 3 & 0 & 27 (4.36) & $\mathrm{N} / \mathrm{A}$ & $\mathrm{N} / \mathrm{A}$ \\
\hline M. haemophilum & 3 & 1 & $36.33(2.08)$ & N/A & $\mathrm{N} / \mathrm{A}$ \\
\hline M. abscessus & 9 & 1 & $4.75(1.78)$ & $\mathrm{N} / \mathrm{A}$ & $\mathrm{N} / \mathrm{A}$ \\
\hline $\mathrm{V} / \mathrm{A}$ - not applicable du & cier & $n$ & rs or incalcula & & \\
\hline
\end{tabular}

Table 3: Antimycobacterial susceptibility patterns of 209 MTBC isolates.

\begin{tabular}{|c|c|c|c|}
\hline \multirow{2}{*}{ Drug } & \multicolumn{2}{|c|}{ \% susceptibility (total number) } & \multirow[b]{2}{*}{$P$-value } \\
\hline & Blood & $\mathrm{BM}$ & \\
\hline Isoniazid & & & \\
\hline Susceptible & 89.67 (165) & $84(21)$ & 0101 \\
\hline $\begin{array}{l}\text { Resistant } \\
\text { Rifampicin }\end{array}$ & $10.33(19)$ & $16(4)$ & 0.491 \\
\hline $\begin{array}{l}\text { Susceptible } \\
\text { Resistant }\end{array}$ & $\begin{array}{c}95.65(176) \\
4.35(8)\end{array}$ & $\begin{array}{c}100(25) \\
0(0)\end{array}$ & 0.599 \\
\hline Ethambutol & & & \\
\hline $\begin{array}{l}\text { Susceptible } \\
\text { Resistant }\end{array}$ & $\begin{array}{c}100(184) \\
0(0)\end{array}$ & $\begin{array}{c}100(25) \\
0(0)\end{array}$ & 1.000 \\
\hline
\end{tabular}

Table 4: Antimycobacterial susceptibility patterns of $35 \mathrm{MAC}$ isolates.

\begin{tabular}{cccc}
\hline \multirow{2}{*}{ Drug } & \multicolumn{2}{c}{$\%$ susceptibility (total number) } & \multirow{2}{*}{$P$-value } \\
\cline { 2 - 3 } & \multicolumn{1}{c}{ Blood } & BM & \\
\hline Clarithromycin & & $55.56(5)$ & 0.027 \\
$\quad$ Susceptible & $92.31(24)$ & $44.44(4)$ & \\
$\quad$ Resistant & $7.69(2)$ & $0(0)$ & 1.000 \\
Moxifloxacin & $7.69(2)$ & $100(9)$ & \\
$\quad$ Susceptible & $92.31(24)$ & & \\
$\quad$ Resistant & & $0(0)$ & 0.153 \\
Linezolid & $26.92(7)$ & $100(9)$ & \\
$\quad$ Susceptible & $73.08(19)$ & & \\
Resistant &
\end{tabular}

dilution. A total of $29(82.86 \%)$ isolates were susceptible to clarithromycin, $33(94.29 \%)$ isolates and $28(80 \%)$ isolates were resistant to moxifloxacin and linezolid, respectively. Percentages of susceptibility of each drug were shown in Table 4. BM isolates (44.44\%) were significantly more resistant to clarithromycin than the blood isolates (7.69\%). There was significant association between sample type and susceptibility frequency to clarithromycin for MAC ( $p=0.027$, Table 4$)$.

\section{DISCUSSION}

In our study, a higher isolation rate of MTBC and MAC (includes $M$. avium, $M$. intracellulare and other $M$. avium complex) was shown in both blood and BM samples similar to our previous study (Foongladda et al., 2009). Surprisingly, unlike before 2010 when no M. abscessus was isolated from any of our blood specimens (Foongladda et al., 2009), M. abscessus was the second most common NTM isolated from both blood and bone marrow in this study. Unfortunately, the outbreak of $M$. abscessus blood infection in 2013 was not detected until this study. Therefore, the source of this outbreak was unknown. This outbreak might be caused by injections of contaminated medical devices and drinking water system. However, evidences were absent and further investigations are needed. In fact, infections caused by $M$. abscessus in Thailand have been previously limited to only local infections such as skin infections and lymphadenitis (Sungkanuparph et al., 2003; 
Phowthongkum et al., 2005). Systemic infection caused by $M$. abscessus in Thailand was considered very rare as only one such was evident in an extensive review of a large teaching Thai hospital, King Chulalongkorn Memorial Hospital during 1997-2003 (Phowthongkum et al., 2005). The high prevalence of $M$. abscessus in this study is also in contrast to a meta-analysis in Iran which reported that $M$. simiae, $M$. terrae, $M$. avium-intracellulare were the most $(44.2 \%)$, second most $(18.3 \%)$, and third most (16.5\%), respectively (Khaledi et al., 2016). This suggests that the high prevalence of $M$. abscessus in Thailand might be unique to this part of Asia. The rise of M. abscessus infection was speculated by some studies to be due to either declining worldwide use of the Bacille Calmette-Guérin vaccine (Sabin et al., 2017) or an emergence of a novel adult-onset immunodeficiency disorder (Lee et al., 2015). However, these require further investigations before any solid conclusion to be made.

As expected, the average time to positivity for those slow growers was longer than the rapid growers. For example, it took 25.7 days for MTBC to be detected by the BACTEC FX system while only 4.75 days were required for $M$. abscessus to have such positivity. Comparing to the previous study from Brazil (Oplustil et al., 2001), our average time to positivity for MTBC and MAC was slightly longer (24 versus 26.4 days for MTBC and 15 versus $17.38-19$ days for MAC). This slight difference might be caused by different model of the blood culture system as the previous study use the BACTEC 460 TB, a radiometric detection, comparing to our BACTEC FX, a fluorometric detection. Other factors can also affect for example, volume of blood and loading of organisms that is different among patients. Though a direct comparison between both systems was not available, a meta-analysis of BACTEC MGIT 960, another fluorometric detection system, also showed slight superior sensitivity $(85.8 \%)$ of the 460 TB system to the $81.5 \%$ sensitivity of the MGIT system (Cruciani et al., 2004).

Similar to a previous study of MTBC (Chaiprasert et al., 2013), the drug resistance was most commonly found with isoniazid $(11 \%)$ and second most commonly found in rifampicin $(3.82 \%)$. The result of MDR-TB in our study was $2.87 \%$. However, these resistance figures were only half of the previous report (Chaiprasert et al., 2013). This might be due to the fact that our MTBC was isolated from blood but MTBC of the previous study were isolated from sputum. This was supported by another report from Thailand showing lower drug-resistance rate of extrapulmonary MTBC isolates than that of pulmonary MTBC isolates (Boonsarngsuk et al., 2018).

For MAC, the most and second most common drug resistance was found in moxifloxacin (94.29\%) and linezolid (80\%), respectively. This is in contrast to a recent study of blood MAC isolates from Taiwan showing that only $21 \%$ were resistant to moxifloxacin and more than $90 \%$ of the isolates were susceptible to linezolid (Lee et al., 2017). However, the high susceptibility to clarithromycin, the first-line drug for MAC treatment, was found in both ours and the Taiwan study. Interestingly, significantly higher rate of clarithromycin resistance was found in BM MAC isolates but the number of BM isolates was small. As a specific correlation between BM and mycobacterial susceptibility has never been reported, more BM MAC isolates are needed before drawing any conclusion.

\section{CONCLUSION}

This study provides average time to positivity of blood and BM cultures for both slow and rapid growers. Moreover, specific patterns of species distribution and antimycobacterial susceptibility pattern of blood and BM isolates were also reported. These data will benefit clinical practitioners for antimycobacterial drug selection while mycobacterial blood or BM culture is pending.

\section{ACKNOWLEDGEMENTS}

We thank Mycobacteriology and Mycology Laboratory, Department of Microbiology, Faculty of Medicine Siriraj Hospital, Mahidol University for providing the blood culture data and result of drugs susceptibility testing.

\section{REFERENCES}

Boonsarngsuk, V., Mangkang, K. and Santanirand, P. (2018). Prevalence and risk factors of drug-resistant extrapulmonary tuberculosis. The Clinical Respiratory Journal 12, 2101-2109.

Bouza, E., Martin-Scapa, C., de Quirós, J. B., Martínez-Hernández, D., Menarguez, J., GómezRodrigo, J., Cosín, J. and Sagues-Cifuentes, M. (1988). High prevalence of tuberculosis in AIDS patients in Spain. European Journal of Clinical Microbiology and Infectious Diseases 7, 785-788.

Chaiprasert, A., Srimuang, S., Tingtoy, N., Makhao, N., Sirirudeeporn, P., Tomnongdee, N., Theankeaw, O., Charoensook, S., Leechawengwongs, M. and Prammananan, T. (2013). Eleven-year experience on anti-TB drugs direct susceptibility testing from Siriraj Hospital, Thailand. Diagnostic Microbiology and Infectious Disease 77, 241-244.

Chin, D. P., Reingold, A. L., Stone, E. N., Vittinghoff, E., Horsburgh Jr, C. R., Simon, E. M., Yajko, D. M., Keith Hadley, W., Ostroff, S. M. and Hopewell, P. C. (1994). The impact of Mycobacterium avium complex bacteremia and its treatment on survival of AIDS patients-a prospective study. Journal of Infectious Diseases 170, 578-584.

Clinical and Laboratory Standards Institute. (2011). Susceptibility testing of mycobacteria, nocardia, and other aerobic actinomycetes-second edition: Approved standard Document M24-A2. CLSI Wayne, PA, USA.

Cruciani, M., Scarparo, C., Malena, M., Bosco, O., Serpelloni, G. and Mengoli, C. (2004). Meta-analysis of BACTEC MGIT 960 and BACTEC 460 TB, with or without solid media, for detection of mycobacteria. Journal of Clinical Microbiology 42, 2321-2325.

Foongladda, S., Pholwat, S., Eampokalap, B., Kiratisin, P. and Sutthent, R. (2009). Multi-probe 
real-time PCR identification of common Mycobacterium species in blood culture broth. The Journal of Molecular Diagnostics 11, 42-48.

Foongladda, S., Roengsanthia, D., Arjrattanakool, W., Chuchottaworn, C., Chaiprasert, A. and Franzblau, S. (2002). Rapid and simple MTT method for rifampicin and isoniazid susceptibility testing of Mycobacterium tuberculosis. The International Journal of Tuberculosis and Lung Disease 6, 1118-1122.

Fuller, D. D., Davis, T. E., Denys, G. A. and York, M. K. (2001). Evaluation of BACTEC MYCO/F Lytic medium for recovery of mycobacteria, fungi, and bacteria from blood. Journal of Clinical Microbiology 39, 2933-2936.

Gangadharam, P. R. and Jenkins, P. A. (1997). Mycobacteria: I Basic Aspects. Springer US.

Jorakate, P., Higdon, M., Kaewpan, A., Makprasert, S., Yuenprakhon, S., Tawisaid, K., Dejsirilert, S., Whistler, T. and Baggett, H. C. (2015). Contribution of the BacT/Alert MB Mycobacterium bottle to bloodstream infection surveillance in Thailand: added yield for Burkholderia pseudomallei. Journal of Clinical Microbiology 53, 910-914.

Kent, P. T. (1985). Public Health Mycobacteriology. Guide for the Level III Laboratory. US Department of Health and Human Services, Public Health Service, Centers for Disease Control.

Khaledi, A., Bahador, A., Esmaeili, D. and Ghazvini, K. (2016). Prevalence of Nontuberculous Mycobacteria (NTM) in Iranian clinical specimens: Systematic review and meta-analysis. Journal of Medical Bacteriology 29-40.

Lee, M. R., Chien, J. Y., Huang, Y. T., Liao, C. H., Shu, C. C., Yu, C. J. and Hsueh, P. R. (2017). Clinical features of patients with bacteraemia caused by Mycobacterium avium complex species and antimicrobial susceptibility of the isolates at a medical centre in Taiwan, 2008-2014. International Journal of Antimicrobial Agents 50, 35-40.

Lee, M. R., Sheng, W. H., Hung, C. C., Yu, C. J., Lee, L. N. and Hsueh, P. R. (2015). Mycobacterium abscessus complex infections in humans. Emerging Infectious Disease 21, 1638-1646.

Oplustil, C. P., Leite, O. H., Oliveira, M. S., Sinto, S. I., Uip, D. E., Boulos, M. and Mendes, C. F. (2001). Detection of mycobacteria in the bloodstream of patients with acquired immunodeficiency syndrome in a university hospital in Brazil. Brazilian Journal of Infectious Diseases 5, 252-259.

Phowthongkum, P., Prasanthai, V., Udomsantisook, N. and Suankratay, C. (2005). Rapidly growing mycobacteria in King Chulalongkorn Memorial Hospital and review of the literature in Thailand. Journal of the Medical Association of Thailand 88, 1153-1162.

Sabin, A. P., Ferrieri, P. and Kline, S. (2017). Mycobacterium abscessus complex infections in children: A Review. Current Infectious Disease Reports 19, 46.

Scarparo, C., Ricordi, P., Ruggiero, G. and Piccoli, P. (2004). Evaluation of the fully automated BACTEC
MGIT 960 system for testing susceptibility of Mycobacterium tuberculosis to pyrazinamide, streptomycin, isoniazid, rifampin, and ethambutol and comparison with the radiometric BACTEC 460TB method. Journal of Clinical Microbiology 42, 11091114.

Sungkanuparph, S., Sathapatayavongs, B. and Pracharktam, R. (2003). Infections with rapidly growing mycobacteria: Report of 20 cases. International Journal of Infectious Diseases 7, 198205.

Tortoli, E., Mariottini, A. and Mazzarelli, G. (2003). Evaluation of INNO-LiPA MYCOBACTERIA v2: Improved reverse hybridization multiple DNA probe assay for mycobacterial identification. Journal of Clinical Microbiology 41, 4418-4420. 